\title{
Intravenous Immunoglobulin Suppresses \\ Chemotherapy-induced Peripheral Neurotoxicity via Macrophage Modulation in Rats and Mice
}

Jun Tanaka ( $\square$ tanaka-jun@jbpo.or.jp )

Japan Blood Products Organization

Masahiko Kajii

Japan Blood Products Organization

\section{Research Article}

Keywords: Chemotherapy-induced peripheral neurotoxicity (CIPN) , Intravenous Immunoglobulin, bortezomib

Posted Date: August 24th, 2021

DOI: https://doi.org/10.21203/rs.3.rs-834697/v1

License: (c) (i) This work is licensed under a Creative Commons Attribution 4.0 International License.

Read Full License 


\section{Abstract}

Chemotherapy-induced peripheral neurotoxicity (CIPN) is a serious adverse effect that leads to treatment discontinuation by patients receiving anticancer therapy. Treatment discontinuation is a serious and lifethreatening problem for patients with cancer; hence, there is a need for drugs that suppress the induction of CIPN by anticancer drugs. Intravenous immunoglobulin (IVIg) reportedly suppresses bortezomibinduced CIPN in a rat model. Here, using rodent models, we showed that IVIg can also suppress peripheral neurotoxicity induced by anticancer drugs other than bortezomib. Furthermore, the suppressive effect of IVIg disappeared when macrophages were depleted. These findings indicate two novel independent possibilities of alleviating CIPN using IVIg. First, CIPN can be controlled by macrophage modulation. Second, IVIg directly combined with anti-cancer drugs can avoid restrictions on the use of anti-cancer drugs due to CIPN inductions. However, further research is necessary for the bench-to-bedside translation of these novel applications of IVIg. Our findings lay a strong foundation for research on IVIg therapeutics.

\section{Introduction}

Patients with anticancer chemotherapy-induced peripheral neurotoxicity (CIPN) experience compromised daily life activities and lowered quality of life post treatment ${ }^{1-3}$. Consequently, treatment must be interrupted even if a patient is responding well to a drug. This causes undue psychological stress in patients who must deal with CIPN-related symptoms and effective drug discontinuation. Although some drugs have been approved to treat CIPN (such as duloxetine, pregabalin, and tricyclic antidepressants), there is no drug that shows a remarkable effect. Moreover, the pathogenesis of CIPN remains unclear ${ }^{4}$.

Intravenous immunoglobulin (IVIg) was developed as a drug for patients with antibody deficiency ${ }^{5}$; however, currently, IVIg is also used to treat many autoimmune disorders, such as Kawasaki disease and idiotypic immune thrombocytopenic purpura ${ }^{6,7}$. The proposed mechanisms underlying IVIg action in autoimmune diseases include the modulation of Fc receptors, interference with the cytokine network and complement proteins, provision of anti-idiotypic antibodies, suppression of lymphocyte effector function $6,8,9$, and presentation of regulatory $T$ cell-inducing epitope termed Tregitope ${ }^{10}$. Additionally, sialylated immunoglobulin $\mathrm{G}(\mathrm{IgG})$ contained in IVIg indirectly upregulates the inhibitory IgG Fc receptor FcyRIIB on effector macrophages ${ }^{7}$. Although IVIg has several functions and is effective against many diseases, its precise action mechanism remains unclear.

Recently, Meregalli et al. reported that IVIg exerts suppressive effects on bortezomib-induced CIPN in rat models ${ }^{11}$. Additionally, they observed that IVIg reduced the infiltration of M1 macrophages into the peripheral nerves. To the best of our knowledge, their study is the first to report the effectiveness of IVIg in alleviating CIPN. However, IVIg is generally not effective for CIPN, because CIPN can also be caused by anticancer drugs other than bortezomib. Recently, our group focused on the multi-functional effects of IVIg and studied the mechanisms underlying the effects under various conditions such as infectious diseases ${ }^{12-15}$, recurrent pregnancy loss ${ }^{16}$, and neurological disease ${ }^{17,18}$. In the present study, we 
focused on whether the suppressive effects of IVIg can be extended to peripheral neurotoxicity induced by other chemotherapy drugs and whether the reduced infiltration of macrophages observed in response to IVIg administration is involved in alleviating CIPN. Therefore, we aimed to clarify the effects of IVIg on peripheral neurotoxicity induced by chemotherapeutics and studied the role of macrophages in IVIgmediated suppression of CIPN.

\section{Results}

\section{IVIg suppresses peripheral neurotoxicity induced by chemotherapeutics other than bortezomib}

We examined the effects of IVIg on peripheral neurotoxicity induced by drugs other than bortezomib using rodents. We studied the effect of IVIg on paclitaxel-induced peripheral neurotoxicity (PIPN) and doxorubicin-induced peripheral neurotoxicity (DIPN) in rats. Our results showed that IVIg alleviated mechanical allodynia in PIPN (Fig. 1a) and DIPN (Fig. 1b) rats. Additionally, in the mouse PIPN model, IVIg alleviated mechanical allodynia (Fig. 2a) and heat hyperalgesia (Fig. 2b).

\section{Role of macrophages in IVIg action to alleviate CIPN}

To clarify the role of macrophages in the action of IVIg, we investigated the influence of macrophage depletion on the suppressive effects of IVIg in the rat PIPN model. Interestingly, IVIg failed to suppress PIPN in the rat model when macrophages were depleted (Fig. 3).

\section{Discussion}

Here, to the best of our knowledge, we first established that IVIg exerts suppressive effects on both PIPN and DIPN. Briefly, we have shown that IVIg suppresses PIPN and DIPN via macrophage modulation using rats or mice. Meregalli et al. reported that IVIg exerts a similar effect on bortezomib-induced peripheral neurotoxicity ${ }^{11}$. These results indicate that IVIg could have a suppressive effect on peripheral neurotoxicity caused by anticancer drugs (regardless of drug types, such as platinum compounds, antitubulins, thalidomide, and bortezomib) ${ }^{1,2}$. However, the mechanism of CIPN induction varies with the anticancer drug. Thus, IVIg does not directly interfere with anticancer drugs and may act on a factor, which is common in the CIPN pathway.

If IVIg can be proven to suppress CIPN, it can be used in combination with anticancer drugs that are effective in patients with CIPN. As IVIg does not cause serious adverse effects and is a multifunctional drug, combination therapy is a rational strategy. Moreover, IVIg is a polyclonal preparation comprising antibodies pooled and concentrated from the plasma of thousands of healthy individuals. Thus, they are highly effective as an immune replacement therapy in immunocompromised patients with cancer receiving chemotherapy. Therefore, IVIg administration could prove beneficial owing its dual roles of suppressing CIPN and alleviating immune deficiency caused by chemotherapy. 
Notably, our results showed that in the absence of macrophages, IVIg loses its CIPN-suppressive effect. Macrophages are the key coordinators of the immune system. Furthermore, IVIg can normalize immune system-related abnormalities. Thus, we speculate that the immunomodulatory effects of IVIg are mediated by macrophages. Although the development of PIPN after macrophage depletion remains unclear, it is possible that in our study, clodronate failed to completely deplete the macrophages in the peripheral neurons; therefore, further exploratory investigations are required. Moreover, the subset of macrophages essential for IVIg action may differ from those required for the onset of PIPN. Nevertheless, based on our results, we can conclude that macrophages are essential for IVIg action.

Our study outcomes emphasize a strong relationship between IVIg action and macrophages. Interestingly, we also confirmed the same phenomenon in a model of recurrent miscarriage, a condition completely different from CIPN. In a model of recurrent miscarriage ${ }^{16}$ depleting macrophages eliminated the effect of IVIg (Supplementary Fig. 1). Thus, the action of IVIg may not change with the model. Moreover, in our previous study in a mouse model of chronic inflammatory demyelinating polyneuropathy, we found that IVIg can modulate macrophage levels ${ }^{17}$. IVIg reportedly exhibits immunostimulatory effects in infectious diseases and an immunosuppressive effect in autoimmune diseases. These seemingly contradictory effects can be explained by understanding how IVlg modulates macrophages, which are the key coordinators of the immune system. Thus, IVIg may act by normalizing the immune system via macrophage modulation. Thus, our study provides new insights to elucidate the mechanisms underlying the dual roles of IVIg.

Here, we demonstrated two distinct and important possibilities of therapeutic application of IVIg suppression in CIPN. First, we found that IVIg acts on macrophages to normalize immunity. Thus, our study further validates that macrophages could be a new target for the development of novel multifunctional drugs. Second, our results showed that a combination therapy may be developed using IVIg to treat cancer and reduce adverse effects. To date, all studies in this field have focused on the direct effects of drugs on cancer. The range of cancer therapy will expand in the future if IVIg is found useful in combination therapy with drugs that are effective in combating cancer. We expect to examine this possibility in the future to expedite therapeutic strategies for cancer patients.

Finally, our data will help to elucidate the action mechanism of IVIg, and its future therapeutic applications. Moreover, to our knowledge, the current therapeutic guidelines for CIPN do not include IVIg therapy. In the present study, we conducted experiments only on rodents, but further studies in other animals are needed to verify the effectiveness of IVIg therapy in alleviating CIPN, and our study emphasizes this urgent need.

\section{Materials And Methods}

\section{Laboratory animals and code of ethics}


Crl:CD (SD) male rats (7 weeks) and Crl:CD1 (ICR) male mice (6 weeks) were purchased from Charles River Japan, Inc. (Kanagawa, Japan). The rats were maintained under specific pathogen-free conditions and a 12-h light/dark cycle; they were used in experiments after 7 weeks of age. All animal procedures were approved by the Animal Care and Use Committee of Japan Blood Products organization. The study was performed in accordance with the guidelines of the Animal Care and Use Committee of Japan Blood Products Organization, which conforms to the policies of the Japan Health Sciences Foundation (The business of the "Japan Health Sciences Foundation" was taken over by the "Japan Pharmaceutical Information Center (https://www.japic.or.jp/calac/english.html)" on 2021 / mar / 31.). This study is reported in accordance with ARRIVE guidelines (https://arriv eguid elines. org).

\section{Induction of CIPN model}

Paclitaxel (Bristol-Myers Squibb, Princeton, NJ USA) was intraperitoneally injected into rats $(1 \mathrm{~g} / \mathrm{kg}$ on days $0,1,2,3,4,7,8,9,10,11,14$, and 15 ) or mice ( $3 \mathrm{mg} / \mathrm{kg}$ on days $0,1,2,3$, and 4 ) to induce PPIN. Paclitaxel was dissolved in 8.3\% Cremophor EL (Nacalai Tesque, Kyoto, Japan), 8.3\% ethanol (FUJIFILM Wako Pure Chemical Corporation, Osaka, Japan), and 83.4\% sterile saline (Otsuka Pharmaceutical Factory, Tokushima, Japan).

Doxorubicin (Nippon Kayaku, Tokyo, Japan), an anticancer drug, was intravenously injected into rats (6 $\mathrm{mg} / \mathrm{kg}$ on day 0 ). Control group animals were administered the same amount of the solvent or saline used to dissolve the drugs.

\section{Administration of IVlg}

IVIg (Japan Blood Products Organization, Tokyo, Japan) was intravenously administered on days 0, 7, 14, and 21 at $1 \mathrm{~g} / \mathrm{kg}$ to the rats treated with paclitaxel or doxorubicin and on days 0 and 5 at $1 \mathrm{~g} / \mathrm{kg}$ to mice treated with paclitaxel. Control group animals were administered the same amount of saline.

\section{Depletion of macrophage}

To deplete macrophages, clodronate liposome (Hygieia Bioscience, Osaka, Japan) was intravenously injected on days $2,5,12$, and 19 at $5.6 \mathrm{mg} /$ body.

\section{Mechanical allodynia and heat hyperalgesia}

Neuropathic pain was evaluated using the dynamic test in accordance with a previously published method ${ }^{19,20}$. Animals were acclimated to the corresponding behavioral test environments, and baseline responses were measured before anticancer drug administration. The occurrence of neuropathic signs was monitored after 3, 4, and 5 weeks (rat) or 1 and 2 weeks (mouse) of anticancer drug injection.

Mechanical allodynia was assessed using a 26-g Semmes-Weinstein Von Frey Anesthesiometer (Tactile Test; Muromachi Kikai, Tokyo, Japan). Briefly, the animals were placed in a compartment with a wire mesh bottom, and later, a Semmes-Weinstein monofilament was applied to the plantar surface of their hind paws with a progressive increase in puncture pressure. The sensory threshold was recorded, and the 
mechanical sensitivity was determined by calculating the mean value of 10 repeated applications per footpad. Mechanical measurements were assessed via verification by two experimenters.

Heat hyperalgesia was measured as the latency to express pain-related behavior using a hot plate $\left(54.5^{\circ} \mathrm{C}\right)$. The following behaviors were observed: 1$)$ licking of foot, 2$)$ fluttering of foot, 3 ) raising of foot, 4) jumping, and the latency until one of them was expressed (latency to paw withdrawal) was measured for individual animals.

\section{Statistical Analysis}

Data are expressed as mean \pm standard error of the mean (SEM). The results are representative of two or more independent experiments. Means were compared using Student's $t$-test. First, the control group and the anticancer drug-administered group were tested. After confirming that the model was established, the anticancer drug-administered group and the IVIg-administered group were tested. Statistical analyses were performed using SAS9.3 software (SAS Institute Inc, NC, USA), and results with $\mathrm{P}<0.05$ (two-tailed) indicated significance.

\section{Declarations}

\section{Acknowledgments}

We are grateful to Prof. A. Kawabata and Assistant Prof. M. Tsubota-Matsunami (Kindai University, Japan) for technical support in animal model development. We also thank Editage for English language editing of the manuscript (https://www.editage.com/).

\section{Author contributions}

MK conceived and designed the study and collated and analyzed the data. JT interpreted the data, directed the research, and wrote the manuscript draft.

\section{Competing interest statement:}

The authors declare no competing interests.

\section{Data availability:}

Authors provide adequate assurances that they can comply with the publication's requirements for sharing materials.

\section{References}


1. Brown, M. R., Ramirez, J. D. \& Farquhar-Smith, P. Pain in cancer survivors. British journal of pain 8, 139-153, doi:10.1177/2049463714542605 (2014).

2. De Groot, A. S. et al. Activation of natural regulatory T cells by IgG Fc-derived peptide "Tregitopes"., 112, 3303-3311 https://doi.org/10.1182/blood-2008-02-138073 (2008).

3. Meregalli, C. et al. High-dose intravenous immunoglobulins reduce nerve macrophage infiltration and the severity of bortezomib-induced peripheral neurotoxicity in rats. Journal of neuroinflammation, 15 , 232 https://doi.org/10.1186/s12974-018-1270-x (2018).

4. Nakae, T., Hirayama, F. \& Hashimoto, M. [Neutralizing activity of human immunoglobulin preparation against toxic shock syndrome toxin-1]. Kansenshogaku zasshi. The Journal of the Japanese Association for Infectious Diseases, 76, 195-202 (2002).

5. Nakae, T., Tanaka, J., Nakano, A. \& Ono, Y. Complement-mediated bactericidal effect of antibodies in human intravenous preparation against multi-drug resistant pseudomonas aeruginosa. The Japanese journal of antibiotics, 61, 379-387 (2008).

6. Tanaka, J. et al. Effective concentration of intravenous immunoglobulin for neutralizing PantonValentine leukocidin in human blood. Journal of infection and chemotherapy: official journal of the Japan Society of Chemotherapy, https://doi.org/10.1016/j.jiac.2017.12.023 (2018).

7. Tanaka, J. et al. Complement-mediated bacteriolysis after binding of specific antibodies to drugresistant Pseudomonas aeruginosa: morphological changes observed by using a field emission scanning electron microscope. Journal of infection and chemotherapy: official journal of the Japan Society of Chemotherapy, 16, 383-387 https://doi.org/10.1007/s10156-010-0074-4 (2010).

8. Tanaka, J. et al. Intravenous Immunoglobulin Suppresses Abortion Relates to an Increase in the CD44bright NK Subset in Recurrent Pregnancy Loss Model Mice. Biology of reproduction, 95, 37 https://doi.org/10.1095/biolreprod.116.138438 (2016).

9. Kajii, M. et al. Intravenous immunoglobulin preparation attenuates neurological signs in rat experimental autoimmune neuritis with the suppression of macrophage inflammatory protein 1 alpha expression. Journal of neuroimmunology, 266, 43-48 https://doi.org/10.1016/j.jneuroim.2013.10.011 (2014).

10. Kajii, M. et al. Prevention of excessive collagen accumulation by human intravenous immunoglobulin treatment in a murine model of bleomycin-induced scleroderma. Clinical and experimental immunology, 163, 235-241 https://doi.org/10.1111/j.1365-2249.2010.04295.x (2011).

11. Flatters, S. J. \& Bennett, G. J. Ethosuximide reverses paclitaxel- and vincristine-induced painful peripheral neuropathy., 109, 150-161 https://doi.org/10.1016/j.pain.2004.01.029 (2004).

12. Nieto, F. R. et al. Tetrodotoxin inhibits the development and expression of neuropathic pain induced by paclitaxel in mice., 137, 520-531 https://doi.org/10.1016/j.pain.2007.10.012 (2008).

13. 13. Nakae, T., Tanaka, J., Nakano, A. \& Ono, Y. Complement-mediated bactericidal effect of antibodies in human intravenous preparation against multi-drug resistant pseudomonas aeruginosa. The Japanese journal of antibiotics 61, 379-387 (2008). 
14. 14. Tanaka, J. et al. Effective concentration of intravenous immunoglobulin for neutralizing PantonValentine leukocidin in human blood. Journal of infection and chemotherapy : official journal of the Japan Society of Chemotherapy, doi:10.1016/j.jiac.2017.12.023 (2018).

15. 15. Tanaka, J. et al. Complement-mediated bacteriolysis after binding of specific antibodies to drugresistant Pseudomonas aeruginosa: morphological changes observed by using a field emission scanning electron microscope. Journal of infection and chemotherapy : official journal of the Japan Society of Chemotherapy 16, 383-387, doi:10.1007/s10156-010-0074-4 (2010).

16. 16. Tanaka, J. et al. Intravenous Immunoglobulin Suppresses Abortion Relates to an Increase in the CD44bright NK Subset in Recurrent Pregnancy Loss Model Mice. Biology of reproduction 95, 37, doi:10.1095/biolreprod.116.138438 (2016).

17. 17. Kajii, M. et al. Intravenous immunoglobulin preparation attenuates neurological signs in rat experimental autoimmune neuritis with the suppression of macrophage inflammatory protein 1alpha expression. Journal of neuroimmunology 266, 43-48, doi:10.1016/j.jneuroim.2013.10.011 (2014).

18. 18. Kajii, M. et al. Prevention of excessive collagen accumulation by human intravenous immunoglobulin treatment in a murine model of bleomycin-induced scleroderma. Clinical and experimental immunology 163, 235-241, doi:10.1111/j.1365-2249.2010.04295.x (2011).

19. 19. Flatters, S. J. \& Bennett, G. J. Ethosuximide reverses paclitaxel- and vincristine-induced painful peripheral neuropathy. Pain 109, 150-161, doi:10.1016/j.pain.2004.01.029 (2004).

20. 20. Nieto, F. R. et al. Tetrodotoxin inhibits the development and expression of neuropathic pain induced by paclitaxel in mice. Pain 137, 520-531, doi:10.1016/j.pain.2007.10.012 (2008).

\section{Figures}

Figure 1a

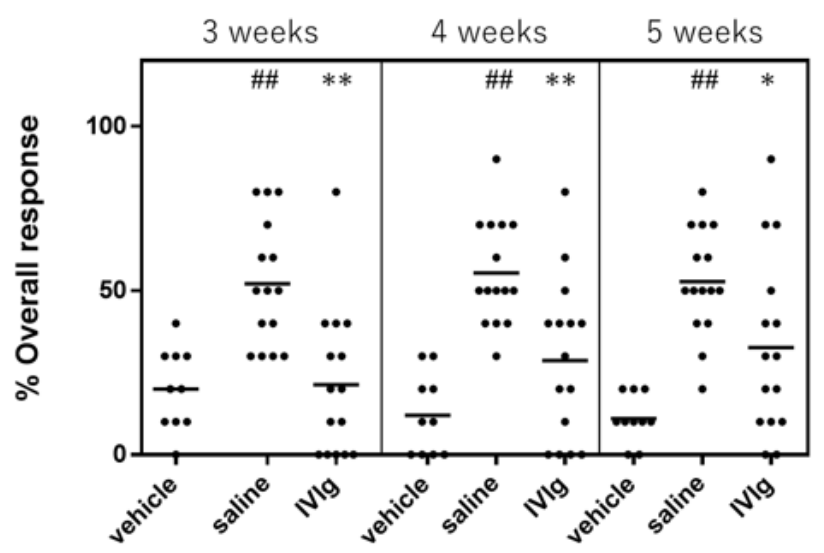

b

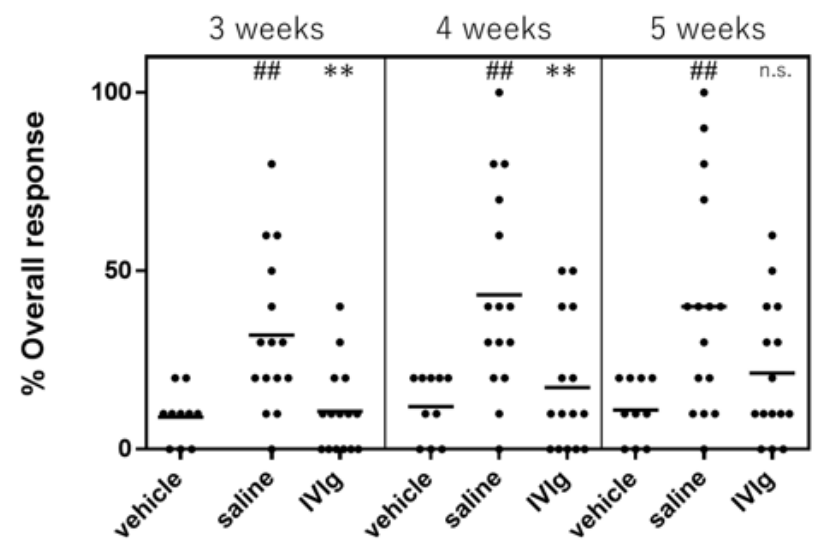




\section{Figure 1}

IVIg suppresses peripheral neurotoxicity induced by antineoplastic drugs other than bortezomib in rat models Representative results of the mechanical allodynia monitoring experiments to evaluate neuropathic signs after 3,4 , and 5 weeks of anticancer drug injection are shown. (a) Rats were intraperitoneally injected with paclitaxel $(1 \mathrm{~g} / \mathrm{kg}$ ) to develop the PIPN model. (b) Rats were intravenously injected with doxorubicin $(6 \mathrm{mg} / \mathrm{kg})$ to develop the DIPN model; IVlg $(1 \mathrm{~g} / \mathrm{kg})$ was intravenously administered on days $0,7,14$, and 21 [data are presented as mean \pm SEM $\{n=10$ (control group) or 15 (DIPN and PIPN groups)\}, \#\#P<0.01 (vs. same time vehicle group by Student's t-test), *P< 0.05 and $* * P<$ 0.01 (vs. same time saline group by Student's t-test), n.s. = not significant]

Figure 2a

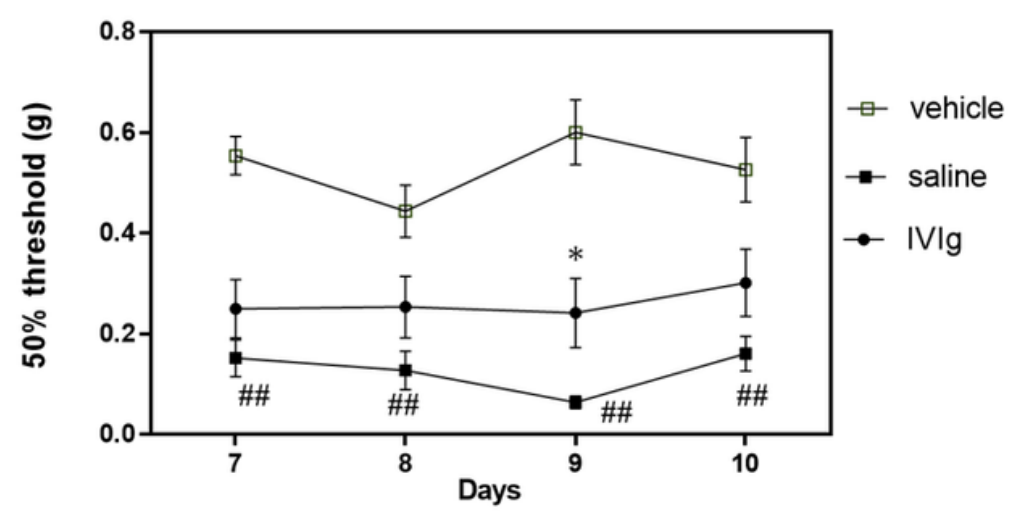

b

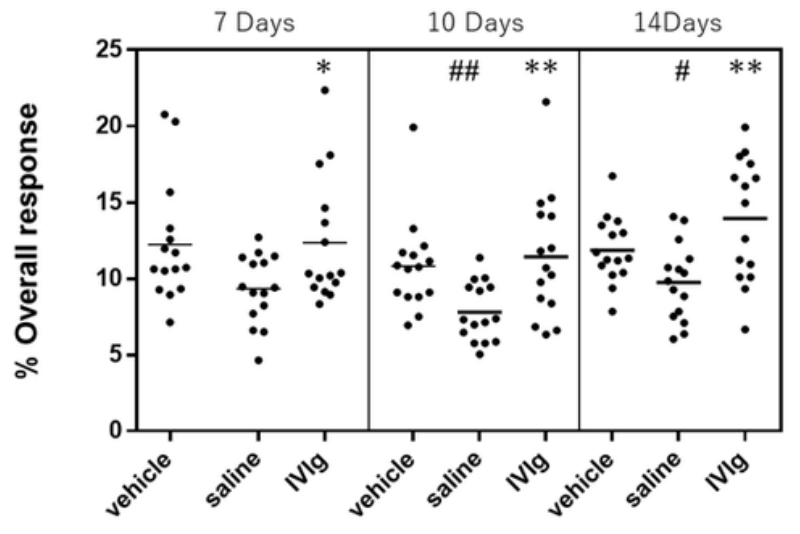

\section{Figure 2}

IVIg suppresses PIPN in mice Representative results of the experiments to monitor the occurrence of neuropathic signs-mechanical allodynia (a) and heat hyperalgesia (b)-after 7, 10, and $14 \mathrm{~d}$ of anticancer drug injection are shown; IVlg $(1 \mathrm{~g} / \mathrm{kg})$ was intravenously administered on days 0 and 5 . Mice were intraperitoneally administered paclitaxel $(3 \mathrm{mg} / \mathrm{kg})$ to induce PIPN [data are presented as mean \pm SEM $(n=15), \# P<0.05$ and \#\#P< 0.01 (vs. same time vehicle group by Student's t-test), ${ }^{*}<0.05$ and $\star \star P<0.01$ (vs. same time saline group by Student's t-test), n.s. = not significant. Results are representative of two or more independent experiments] 
Figure 3

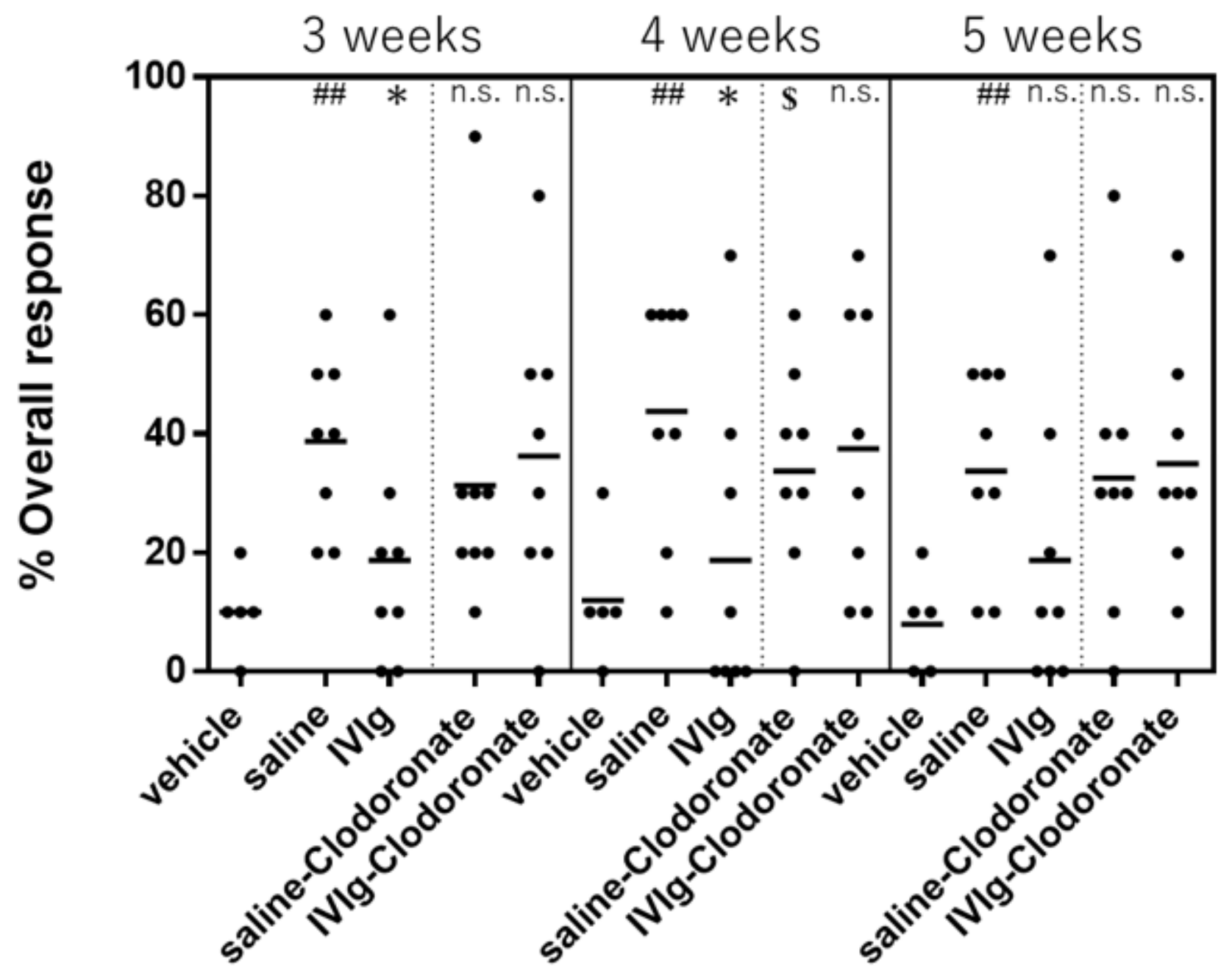

Figure 3

Suppressive effect of IVIg disappeared following macrophage depletion Representative results of the experiments to monitor the occurrence of neuropathic signs of mechanical allodynia after 3,4 , and 5 weeks of anticancer drug injection are shown; $I \mathrm{Vlg}(1 \mathrm{~g} / \mathrm{kg})$ was intraperitoneally administered on days 0 , 7,14 , and 21 ; clodronate liposomes (5.6 mg/body) were intraperitoneally administered $2 \mathrm{~d}$ before the administration of IVlg. Rats were intraperitoneally injected with paclitaxel $(1 \mathrm{~g} / \mathrm{kg})$ to induce PIPN [data are presented as mean \pm SEM $\{\mathrm{n}=5$ (control group) or 8 (non-control groups)\}, \#\#P<0.01 (vs. same time vehicle group by Student's t-test), ${ }^{*} \mathrm{P}<0.05$ (vs. same time saline group by Student's t-test), $\$ \mathrm{P}<0.05$ (vs. same time saline vehicle by Student's t-test), n.s. = not significant. Results are representative of two or more independent experiments]

\section{Supplementary Files}


This is a list of supplementary files associated with this preprint. Click to download.

- SupplementaryFig.1.pptx

- Supplementaryinformation.docx 